\title{
GASRP-FP: A hybrid metaheuristic to solve fuzzy portfolio selection with non-financial goals
}

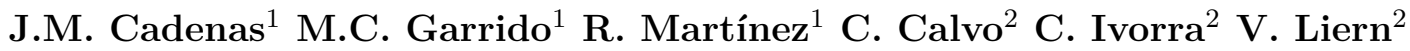 \\ ${ }^{1}$ Dep. of Information Engineering and Communication, University of Murcia, Murcia, Spain \\ jcadenas@um.es, carmengarrido@um.es, raquel.m.e@um.es \\ ${ }^{2}$ Dep. of Mathematics for Economics and Business, University of Valencia, Valencia, Spain \\ clara.calvo@uv.es, carlos.ivorra@uv.es, vicente.liern@uv.es
}

\begin{abstract}
The emergence of more powerful computational techniques has stimulated the development and study of more sophisticated models for portfolio selection problem including further constraints and further financial and non-financial decision criteria. In this paper, we propose a hybrid approach composed of two genetic algorithms to solve a fuzzy portfolio selection model with cardinality constraints, semicontinuous variable and non-financial goals. An analytical description of the efficient frontier and socially responsible solutions are obtained.
\end{abstract}

Keywords: Portfolio selection, Efficient frontier, Socially responsible investment, Genetic Algorithm

\section{Introduction}

The modern portfolio selection problem is a classical model for determining the optimal composition of a portfolio according to an investor's preferences. The model is credited to Markowitz [10], who is rightfully regarded as the founder of modern portfolio theory. This problem is more naturally seen as a bi-objective problem with two financial goals: maximizing the expected return and minimizing the risk of the portfolio. Markowitz considered the return of each asset as a random variable and measured the expected return and the risk of a portfolio by means of the mean of the corresponding weighted sum of random variables and the quadratic form associated to the variance-covariance matrix, respectively. Under normality hypotheses, these can be estimated by taking historical data as a sample. However, finer measures of the risk and the return can be considered $[7,9,11]$.

On the other hand, Markowitz just imposed the trivial non-negativity constraints on the variables together with the sum-one constraint for the weights of the assets in the portfolio. Nevertheless, the emergence of more powerful computational techniques has stimulated the development and study of more sophisticated models including further constraints and further financial and non-financial decision criteria.

A relevant example of non-financial additional investment goals is Socially Responsible Investment
(SRI). SRI is broadly defined as an investment process that integrates not only financial but also social, environmental, and ethical concerns into investment decision making. However, few portfolio selection models including these goals can be found in the literature $([1,2,3,12,14]$ are some examples). One of the main problems faced by those authors is the rating of assets with regard to these issues. This poses two main mathematical problems: how to evaluate the social responsibility of the assets, which is by its nature a vague and imprecise concept, and how to aggregate in a final rating a great amount of relevant but imprecise information about firms and/or funds.

In [6], a model for solving a fuzzy portfolio selection problem with cardinality constraints, semicontinuous variables and non-financial goals is proposed. In a first step, the efficient financial solutions, in terms of risk and return, are obtained as proposed in [5]. Then, in a second step a nonfinancial secondary goal is considered and new solutions are offered to the investor (interesting solutions from a financial point of view are not discarded as a result of the introduction of the non-financial constraints). From a computational viewpoint, the trouble with this approach emerges with medium and large-sized portfolio problems. For small-sized problems, in the first step, the analytical description of the efficient frontier is exact, whereas for mediumsized instances, a reasonable approximation of the exact frontier is provided. Furthermore, the CPUtime increases with the problem size, making the calculation of the solutions unviable for medium and large-sized problems.

In this paper, we describe a Soft-Computing method composed of two genetic algorithms (GAs) to solve this model, which is more adequate for medium and large-sized problems. The rest of the paper is organized as follows. In next section, a measure of the social responsibility of the assets and a formulation of the portfolio selection problem including a non-financial goal are presented. Section 3 describes the method for solving this model. In order to illustrate the proposed method, a example is presented in section 4 . Finally, section 5 includes the conclusions. 


\section{A fuzzy portfolio selection model with non-financial goals}

\subsection{A measurement of the degree of social responsibility}

The main SRI tool is investment in socially responsible mutual funds and the most common SRI strategy is screening, [13]. This investment strategy consists of checking companies for the presence or absence of certain social, environmental, ethical and/or good corporate governance characteristics. However, these characteristics have an ambiguous, imprecise and/or uncertain nature. Therefore, evaluating the social responsibility of the assets is not a simple question. In this context Fuzzy Set Theory offers some elements which can help the experts to assess the degree of social responsibility of a financial asset, [12].

We focus on equity mutual funds. Socially responsible equity mutual funds invest primarily in stocks and the degree of them is measured at two levels [6]: First, the fuzzy social performance of each firm invested in by the mutual fund is evaluated, and second, the quality of the non-financial information provided by the mutual fund managers is evaluated by using the reports of information on companies' performance with respect to environmental, social and governance criteria.

Let us consider $n$ equity mutual funds $\left\{F_{1}, F_{2}, \ldots, F_{n}\right\}$ which invest principally in stocks which are portions of ownership of a corporation or firm. Let us consider a set $\left\{f_{k}\right\}_{k=1}^{q}$ of $q$ firms in which the funds can invest at a certain moment of time. As equity mutual funds invest almost $100 \%$ in stocks, [6], the degree of social responsibility of the funds is evaluated using the degree of social responsibility of the firms they invest in. In order to do so, $m$ social screens $\left\{s_{1}, s_{2}, \ldots, s_{m}\right\}$ are taken into account, which are the usual screens applied by the main rating agencies it is assumed that the expert evaluates the degree of social responsibility of each firm $k$ with respect to each screen $j$ by assigning it an interval:

$$
\tilde{s}_{k j}=\left\{\left(s_{k j},\left[b_{k j}^{L}, b_{k j}^{U}\right]\right), 1 \leq k \leq q, 1 \leq j \leq m\right\}
$$

where $\left[b_{k j}^{L}, b_{k j}^{U}\right] \subseteq[0,1]$. Thus, for each firm, the interval $\left[b_{k j}^{L}, b_{k j}^{U}\right]$ represents the membership function of firm $k$ with respect to the social screen $j$, considered as a tolerance interval. And, the interval representing the social responsibility degree of each firm $k$ is the following:

$$
\left[b_{k}^{L}, b_{k}^{U}\right]=\sum_{j=1}^{m}\left[b_{k j}^{L}, b_{k j}^{U}\right], \quad 1 \leq k \leq q .
$$

Then, taking into account the percentage invested in each firm by each mutual fund, the degree of social responsibility of each mutual fund is:

$$
\left[b_{i}^{L}, b_{i}^{U}\right]=\sum_{k=1}^{q} \alpha_{k}^{i}\left[b_{k}^{L}, b_{k}^{U}\right], \quad 1 \leq i \leq n,
$$

where $\alpha_{k}^{i} \in[0,1]$ represents the weighting percentage of firm $k$ in mutual fund $i$.

Next step consists of obtaining a set of weights for each mutual fund, [6]. These weights play a correcting factor as they represent the degree of quality (in terms of transparency and credibility) of the information on the social screening process provided by the mutual funds' managers (they depend on several criteria: quality of the description of the screening process, existence of an external research team composed of experts in SRI, periodical non-financial audits, description of engagement policy, public disclosure of proxy voting practices and education of the fund manager on SRI practices). Therefore, a weight is assummed each interval:

$$
\tilde{w}_{i}=\left\{\left(w_{i},\left[b_{w_{i}}^{L}, b_{w_{i}}^{U}\right]\right)\right\}, \quad 1 \leq i \leq n .
$$

These weights will be also a discrete set for each mutual fund. The interval represents the membership function of the weight assigned to mutual fund $i$ considered as a tolerance interval. Thus, for each mutual fund $F_{i}$, its Fuzzy Social Responsibility degree, $\widetilde{S R D}_{i}$, is defined as:

$$
\widetilde{S R D}_{i}=\tilde{w}_{i} \tilde{s}_{i}, \quad 1 \leq i \leq n,
$$

and taking into account (1) and (2), its membership function is given, for each $1 \leq i \leq n$, by

$$
\mu_{\widetilde{S R D_{i}}}\left(F_{i}\right)=\left[b_{w_{i}}^{L}, b_{w_{i}}^{U}\right] *\left[b_{i}^{L}, b_{i}^{U}\right]=\left[b_{w_{i}}^{L} b_{i}^{L}, b_{w_{i}}^{U} b_{i}^{U}\right]
$$

Finally, we handle the Fuzzy Social Responsibility degrees by means of their expected values, [6]: For each $1 \leq i \leq n$

$$
E V\left(\widetilde{S R D}_{i}\right)=\frac{1}{2}\left(b_{w_{i}}^{L} b_{i}^{L}+b_{w_{i}}^{U} b_{i}^{U}\right)
$$

\subsection{A multiobjetive model with cardinality constraints and semicontinuous variable}

Our starting point is the following crisp multiobjective portfolio selection model $(\mathrm{CP})$ :

$$
\begin{array}{lll}
\text { Max } & r(x)=e x & \\
\text { Min } & R(x)=x^{t} V x & \\
\text { s.t. } & 1 x=1 & \\
& m \leq \sum_{i} y_{i} \leq M & \\
& l_{i} y_{i} \leq x_{i} \leq u_{i} y_{i}, & i=1, \ldots, n \\
& x_{i} \geq 0, y_{i} \in\{0,1\} & i=1, \ldots, n
\end{array}
$$

where $V$ is the variance-covariance matrix, $e$ is the vector of expected returns, the variables $x_{i}$ represent the weight of each asset in the portfolio (and hence they must satisfy constraint (c1)) and the binary variable $y_{i}$ takes the value 1 if and only if the corresponding asset actually appears in the portfolio. These auxiliary variables allow us to introduce cardinality and semicontinuous variable constraints into the model. Cardinality constraints impose conditions as (c2), bounding the number of assets appearing in the portfolio, but this is just an instance, 
and more particular constraints on specific sets of assets can be introduced in the same way. Semicontinuous variable constraints $(\mathrm{c} 3)$ require the variables $x_{i}$ to be on an interval $\left[l_{i}, u_{i}\right]$ unless they take the value $0,[4]$.

Constraints (c1)-(c3) are usually imposed as diversification constraints and we take them as the hard constraints of our problem, that is, as the constraints that the investor definitely wishes to impose. Furthermore, we consider two objectives: the minimization of the risk and maximization of the return. In particular, we can consider two models derivatives of the model $(\mathrm{CP})$ : a) the first objective as a main goal $(e x \geq r)$ together with the minimization of the risk $R(x)$ (model $\left.\mathrm{CP}_{r}\right)$; and $\mathrm{b}$ ) the second objective as a main goal $\left(x^{t} V x \leq R\right)$ together with the maximization of the return $r(x)$ (model $\mathrm{CP}_{R}$ ).

Recall that the efficient frontier of a portfolio selection problem is the set of efficient (i.e. nondominated) portfolios (pairs of return and risk).

Definition 2.1. Given two portfolios $x_{1}, x_{2}, x_{1}$ dominates $x_{2}$ if $\left(R\left(x_{1}\right)<R\left(x_{2}\right)\right.$ and $\left.r\left(x_{1}\right) \geq r\left(x_{2}\right)\right)$ or $\left(R\left(x_{1}\right) \leq R\left(x_{2}\right)\right.$ and $\left.r\left(x_{1}\right)>r\left(x_{2}\right)\right)$

Definition 2.2. Efficient frontier is the set $P^{*}$ defined as $P^{*}=\left\{x / \nexists x^{\prime}, \quad x^{\prime}\right.$ dominates $\left.x\right\}$

The frontier determines two functions $R_{e f}(r)$ and $r_{e f}(R)$ (defined on suitable intervals) providing the minimum risk we can reach to ensure an expected return greater or equal to $r$ and the maximum expected return we can reach with a risk not greater than $R$, i.e. the optimal values of $\left(\mathrm{CP}_{r}\right)$ and $\left(\mathrm{CP}_{R}\right)$ respectively.

\subsection{Adding fuzzy conditions and non-financial goals}

Now we state a fuzzy problem whose fuzzy constraint set will contain portfolios "not too far" from a given point of the efficient frontier expressing the investor's initial preferences about risk and return, and whose fuzzy goal set will contain portfolios that are "efficient enough" and "good enough" with regard to the non-financial goal, [6]. Then, a fuzzy optimization problem has a fuzzy constraint set $\tilde{C}$, which means that we consider not only feasible solutions and non feasible solutions, but also partially feasible solutions that can be acceptable for the decision-maker to a certain extent. On the other hand, we need a fuzzy goal set $\tilde{G}$, classifying the solutions as good or bad solutions (but accepting partially good solutions). These sets determine a fuzzy decision set $\tilde{D}=\tilde{C} \cap \tilde{G}$, where the fuzzy intersection is usually defined as the fuzzy set determined by the minimum of the corresponding degrees of membership, although several alternatives can be considered. The fuzzy optimal solution of a fuzzy problem is that possessing the best degree of membership of the decision set.
The feasible set $\tilde{C}$ could be $\tilde{C}=\tilde{C}_{r} \cap \tilde{C}_{R}$, where the membership functions of the fuzzy sets $\tilde{C}_{r}$ and $\tilde{C}_{R}$ are given by:

$$
\begin{gathered}
\mu_{\tilde{C}_{r}}(x, y)= \begin{cases}\frac{1}{\frac{r-r_{0}+s_{r}}{s_{r}}} & \text { if } r \geq r_{0}, \\
0 & \text { if } r_{0}-s_{r}<r<r_{0},\end{cases} \\
\mu_{\tilde{C}_{R}}(x, y)= \begin{cases}\frac{1}{R_{0}+s_{R}-R} \\
\frac{R_{R}}{s} & \text { if } R \leq R_{0}, \\
\text { if } R_{0}<R<R_{0}+s_{R},\end{cases}
\end{gathered}
$$

where $r$ and $R$ are respectively the expected return and the risk of the portfolio $(x, y)$ and the values $r_{0}$, $R_{0}, s_{r}$ and $s_{R}$ are determined from the investor's preferences. $r_{0}$ and $R_{0}$ are an expected return and a risk that the investor considers as completely acceptable, but he/she would accept worse values until reaching the tolerances $s_{r}$ and $s_{R}$ if this provides better results for the non-financial goal.

Next we define a fuzzy goal set $\tilde{G}$ from two auxiliary fuzzy sets $\tilde{E}$ and $\tilde{S}$, the first one defining the "efficient enough" portfolios and the second one defining the "good enough" ones with regard to the non-financial goal (always according to the investor's preferences). The set $\tilde{E}$ will express what we are loosing by accepting a non-efficient portfolio, and so efficient portfolios will be now the totally efficient portfolios, i.e. those having degree of membership of $\tilde{E}$ equal to 1 .

First we define efficiency with regard to the expected return and then, the efficiency with regard to the risk by means of two fuzzy sets $\tilde{E}_{r}$ and $\tilde{E}_{R}$. The membership of $\tilde{E}_{r}$ is:

$$
\mu_{\tilde{E}_{r}}(x, y)= \begin{cases}1-\frac{r_{\mathrm{ef}}(R)-r}{t_{r}} & \text { if } r \geq r_{e f}(R)-t_{r} \\ 0 & \text { otherwise }\end{cases}
$$

where $t_{r}$ is a tolerance determined from the investor's preferences and $r_{\text {ef }}(R)$ is the efficient expected return corresponding to the risk $R$ of the portfolio. This means that the degree of efficiency with regard to the expected return reaches the value 0 when the difference between the expected return $r$ of the portfolio and $r_{\text {ef }}(R)$ exceeds a tolerance fixed by the investor.

Analogously, we define the membership function of $\tilde{E}_{R}$ as

$\mu_{\tilde{E}_{R}}(x, y)= \begin{cases}1-\frac{R-R_{\mathrm{ef}}(r)}{t_{R}} & \text { if } R \leq R_{e f}(r)+t_{R}, \\ 0 & \text { otherwise }\end{cases}$

that is, the degree of efficiency of a portfolio with regard to the risk is 1 for efficient portfolios and reaches the value 0 when the difference between the risk $R$ of the portfolio and the efficient risk $R_{\mathrm{ef}}(r)$ for its return $r$ exceeds a given tolerance $t_{R}$.

Now we define $\tilde{E}=\tilde{E}_{r} \cap \tilde{E}_{R}$, where the membership function of the fuzzy intersection is defined as the minimum of the previously defined membership functions. Hence the set $\tilde{E}$ allows us to speak about 
partially efficient portfolios in such a way that efficient portfolios in the usual sense are now the totally efficient ones, but a portfolio close enough to the efficient frontier is considered as "almost efficient" in the fuzzy sense.

At this point we introduce the investor's preferences on the secondary goal. Let us assume that these preferences are given by a fuzzy set $\tilde{S}$, i.e. the membership degree of $\tilde{S}$ indicates "how good" a given portfolio is with regard to the secondary goal. When the secondary goal is SRI, we can define the Social Responsibility Degree of a portfolio as

$$
\operatorname{SRD}(x)=\sum_{i=1}^{n} E V\left(\widetilde{S R D}_{i}\right) x_{i}
$$

and then normalize it as

$$
\mu_{\tilde{S}}(x, y)=\frac{\operatorname{SRD}(x)}{\operatorname{MSRD}},
$$

where MSRD is the maximum value that $\operatorname{SRD}(x)$ attains on the set $X$, which can be calculated by solving a linear problem.

Then we define our fuzzy goal set $\tilde{G}$ by means of the membership function as a weighted sum

$$
\mu_{\tilde{G}}(x, y)=w \mu_{\tilde{S}}(x, y)+(1-w) \mu_{\tilde{E}}(x, y),
$$

where the weight $w$ expresses the importance of the secondary goal for the investor with regard to efficiency. So, a high value for $w$ means that the investor is willing to go relatively far from the efficient frontier in order to obtain higher values of $\mu_{\tilde{S}}$, whereas a small value of $w$ means that the investor wishes to stay near the efficient frontier. In any case, recall we have defined the feasible set in such a way that only good enough solutions with regard to the financial goals are under consideration, and so the financial goals are always the main goals of the problem. More specifically, a large value for $w$ means that, among the acceptable solutions with regard to the financial goals, those best with regard to $\tilde{S}$ are preferred, and only for similar values with regard to $\tilde{S}$ the degree of efficiency becomes relevant.

All in all, the degree of membership of the decision set is given by

$\mu_{\tilde{D}}(x, y)=\min \left\{\mu_{\tilde{C}}(x, y), w \mu_{\tilde{S}}(x, y)+(1-w) \mu_{\tilde{E}}(x, y)\right\}$

and the fuzzy model with non-financial goals (FP) is the problem determined by this decision set, whose optimal solutions are those with maximum degree of membership of $\tilde{D}$ :

Max. $\min \left\{\mu_{\tilde{C}}(x, y), w \mu_{\tilde{S}}(x, y)+(1-w) \mu_{\tilde{E}}(x, y)\right\}$

s.t. $1 x=1$

$$
m \leq \sum_{i} y_{i} \leq M
$$

$l_{i} y_{i} \leq x_{i} \leq u_{i} y_{i}, \quad i=1, \ldots, n$

$x_{i} \geq 0, y_{i} \in\{0,1\} \quad i=1, \ldots, n$
As we can see in this model, the objective function needs the explicit form of the efficient frontier in order to obtain the values $r_{e f}(R)$ and $R_{e f}(r)$ corresponding to the risk $R$ and return $r$, respectively. This efficient frontier, under the conditions proposed in the set $\mathrm{X}$, consists of a piecewise continuous function defined by a finite number of arcs of parabola. The terms of cardinality and bounded variables make the efficient frontier becomes more complicated.

\section{A hybrid metaheuristic}

A portfolio selection problem can turn into a more complex problem depending on the constraints that are added to the problem (the more irregular the efficient frontier is, the more computational efforts are required). To solve the problems with conventional methods, a high computational effort is required in medium/large-sized and complex problems. SoftComputing methods try to solve the drawbacks of the conventional methods, and they can work with less computational effort with these problems.

We propose a hybrid metaheuristic (named GASRP-FP) for solving the model (FP) in order to obtain the socially responsible portfolios. For this, firstly the explicit efficient frontier and subefficient frontiers have to be calculated, and then the socially responsible portfolios are obtained taking into account the constraints and the parameters of the problem. Before going any further, it is important to notice that after obtaining the socially responsible portfolios, we can offer to the investor, not only the best option taking the result of the efficient frontier, but also, a subefficient portfolios near the efficient frontier from the viewpoint of their social responsibility.

The proposed metaheuristic is composed of two steps: The first step consists of a multiobjective GA of second generation (NSGA-II type, [8]) and it is responsible for the efficient to obtain explicit frontier (and subefficient frontiers) from a given problem (solutions to the model (CP)); and the second consists of a GA that works in parallel and is executed for each portfolio of the efficient and subefficient frontier, and it is responsible for getting socially responsible portfolios (solutions to the model (FP)).

The metaheuristic scheme is shown in Figure 1.

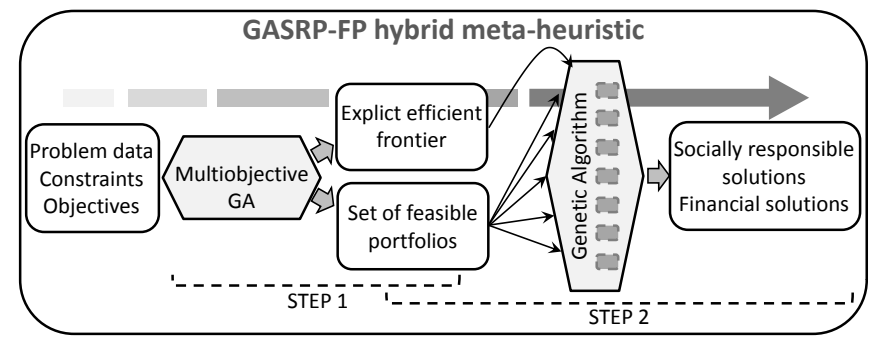

Figure 1: Metaheuristic scheme 


\subsection{Obtaining efficient frontier and feasible portfolios (STEP 1)}

The efficient frontier of the model (CP) is composed of a set of quadratic functions that describes the relation between risk and return, $(R, r)$.

The description of the elements of the multiobjective GA used to obtain the efficient frontier (and subefficient frontiers) of the model (CP) is as follows:

Individual: The individual codification is binary where the individual length is equal to the number of assets $(y)$. Each gene represents an asset and when the gene is equal to zero, the user does not invest in this asset. On the contrary, when the gene is equal to one, the user invests in this asset.

Besides, the individual carries associated some information. Specifically the associated information is: the parameters $(a, b, c)$ of the quadratic function $a r^{2}+b+c=R$, the domain $\left[r_{\min }, r_{\max }\right]$ of quadratic function and the values $L$ and $U$ (lower and upper saturated values). A value is saturated when the value invested in the asset $i$ is exactly $l_{i}$ or $u_{i}$.

Briefly, an individual is defined by the following parameters: $\quad\left[y, a, b, c, r_{\min }, r_{\max }, L, U\right]$ (these parameters are calculated by the procedure KETF described in [5] and included in this GA). It is important to notice that the parameter $y$ is the individual and the rest of parameters are additional information.

Population: The population is composed of the number of non-dominated individual and of the $N$ dominated individuals that give diversity to the problem. The number of non-dominated individual is not limited.

Fitness: The fitness function has a double purpose. On the one hand, the fitness changes the domain of the individual. The aim is to find the dominance of the individual: the function divides the individual and adjusts the domain in order to have dominated and non-dominated individuals. So, the individual that dominates another individual has a fitness equal to one and the dominated one has a fitness equal to zero. On the other hand, besides that dominance value, the fitness function uses a front and niche function in order to discriminate when two individuals have the same dominance value. The aim of this function is to obtain more diversity in the complete domain of the problem. The front and niche function is calculated as follows:

$$
F N=\frac{o_{r}^{i+1}-o_{r}^{i-1}}{o_{r}^{\text {min }}-o_{r}^{\text {max }}}+\frac{o_{R}^{i+1}-o_{R}^{i-1}}{o_{R}^{\text {min }}-o_{R}^{\text {max }}}
$$

where $o_{r}^{i}$ represents the population ordered by return (high to low) and $o_{R}^{i}$ represents the population ordered by risk (low to high).

Selection: The selection operator is elitist (the individuals with dominance value equal to one are saved). To select the best individual of a population, the operator uses a binary tournament function where two individuals are compared considering their dominance value and if these values are equal, the operator returns the individual with the higher value of the front and niche function.

Crossing: The crossover operator is performed by single crossover point. Given two individuals $y_{1}$ and $y_{2}$, the operator considers the number of enabled assets of the two individuals and considers the assets that belong to the sets $L$ and $U$. The crossing is carried out as follows:

Considering as parents:

$$
\begin{aligned}
& y_{1} \rightarrow\left(\sum y_{1 i}=A\right), L_{1}, U_{1} \\
& y_{2}-\rightarrow\left(\sum y_{2 i}=B\right), L_{2}, U_{2}
\end{aligned}
$$

the offsprings are defined as:

$$
\begin{aligned}
& y_{H 1} \rightarrow\left(A \leq \sum y_{H 1 i} \leq B\right), L_{H 1}, U_{H 1} \\
& y_{H 2} \rightarrow\left(A \leq \sum y_{H 2 i} \leq B\right), L_{H 2}, U_{H 2}
\end{aligned}
$$

where $L_{H 1} \cup L_{H 2}=L_{1} \cup L_{2}$ and $U_{H 1} \cup U_{H 2}=U_{1} \cup U_{2}$.

Mutation: The mutation operator is applied by individual and there are several options, that are carried out with the same probability. The options are: To enable and disable an asset, to add or remove an asset of $L$, to add or remove an asset of $U$, to exchange an enabled asset for a disabled asset, to exchange a disabled asset for an enabled asset. Before carrying out a mutation, the constraints of the problem are always tested, and if the mutation failed to fulfill some constraints, the mutation is not performed.

Having presented the elements of this multiobjective GA, the returned solution is a set of nondominated individuals, and these individuals compose the efficient frontier. Furthermore, this algorithm obtains a set of subefficient portfolios. The set of efficient and subefficient portfolios constitutes the set of feasible portfolios from which a set of socially responsible portfolios will be obtained.

Once the set of feasible portfolios is obtained, a GA is used to calculate the best socially responsible portfolios. This GA will be executed for each feasible portfolio (a parallel execution will be carried out). This GA is defined in the next section.

\subsection{Obtaining socially responsible portfolios (STEP 2)}

After obtaining the efficient frontier, we want to calculate other portfolios, near this efficient frontier taking into account their social responsibility. To do that a parallel GA has been developed. The decision to develop a parallel GA is clear, the aim of this parallel GA is to obtain the most socially responsible portfolio for each feasible portfolio taking 
into account the distance to the efficient frontier. So, the GA launches a process in parallel for each possible portfolio.

The input for the algorithm is the output of the algorithm STEP 1. But in this case, the input is only the portfolios composition, ie, the set of portfolios $\left\{y_{1}, y_{2}, \ldots, y_{m}\right\}$ with dominance value equal to one and equal to zero (efficient and subefficient portfolios). Restricting the search domain to which the user has indicated, a portfolio is sought, with its proper investment, in order that the user can get the higher value of degree of social responsibility. The GA components are described as follows:

Individual: The individual codification is real where depending on the portfolio, each gene represents the invest of the corresponding asset and the individual represents the investment vector $x$. Each individual must verify the conditions proposed in the set $X$.

Fitness: The fitness function defines the social responsibility taken into account the distance of the portfolio to the efficient frontier. The greater the fitness function is, the better the portfolio is from a non financial viewpoint. The fitness function is the objective function of the model (FP).

$$
\min \left\{\mu_{\tilde{C}}(x, y), w \mu_{\tilde{S}}(x, y)+(1-w) \mu_{\tilde{E}}(x, y)\right\}
$$

Selection: The selection is performed by binary tournament, being better the individual with greater fitness value.

Crossing: The crossover operator is performed by using a version of the $\alpha$-BLX operator. The crossover operator takes two individuals $c r_{1}$ and $\mathrm{cr}_{2}$ defined by $\left(x_{c r 11}, x_{c r 12}, \ldots, x_{c r 1 n}\right)$ and $\left(x_{c r 21}, x_{c r 22}, \ldots, x_{c r 2 n}\right)$, respectively and calculates the following values:

$$
T a_{i}=a_{i}-\alpha I_{i}, T b_{i}=b_{i}+\alpha I_{i} \text { with } \alpha \in[0,1]
$$

where $T a_{i} \leq l_{i}, T b_{i} \leq u_{i}, a_{i}=\min \left\{x_{c r 1 i}, x_{c r 2 i}\right\}$, $b_{i}=\max \left\{x_{c r 1 i}, x_{c r 2 i}\right\}$ and $I_{i}=b_{i}-a-i$.

Taking $n$ values randomly in the interval $\left[T a_{i}, T b_{i}\right], H P=\left(h p_{1}, h p_{2}, \ldots, h p_{n}\right)$ is built and $H C=\left(l_{1}, l_{2}, \ldots, l_{n}\right)$ where $S=\sum_{i} l_{i}$.

To complete a correct offspring, a random value $h p_{j}$ from HP is taken and $H C_{j}=h p_{j}$ and $S=S+$ $\left(h p_{j}-l_{i}\right)$. This process is carried out until $S>1$ or until having used all $h p_{j}$ values $S<1$. In these situations the $\mathrm{HC}$ values must be modified to fulfill the constraints $\sum_{i} H C_{i}=1$.

Mutation: The mutation operator is applied by individual and has two options. The first option is to exchange the investments between two genes. With this option the constraint that all the investments have to sum one is fulfilled. The second option is to change the investment of a gene and to fulfill the constraint to sum one, adding or removing the difference between other genes.
For each feasible portfolio, the GA returns the degree of social responsability and investment in each asset.

\section{An example of socially responsible portfolio selection}

In order to compare the solutions provided by proposed approach, we will analyze in this section a small real numerical example.

We consider a problem with the 10 funds listed in Table 1, for which we determine the portfolio's environmental responsibility degree $S R D(\cdot)$ based on mutual funds' fuzzy environmental responsibility degrees (see Table 2).

\begin{tabular}{ll}
\hline$\#$ & Name \\
\hline F1 & Calvert Large Cap Growth A \\
F2 & Calvert Social Investment Equity A \\
F3 & Domini Social Equity Inv \\
F4 & Green Century Equity \\
F5 & MMA Praxis Core Stock A \\
F6 & BlackRock Index Equity Inv A \\
F7 & Dreyfus Appreciation \\
F8 & JPMorgan Equity Index Select \\
F9 & Legg Mason Cap Mgmt All Cap B \\
F10 & MFS Blended Res. Core Equity A \\
\hline
\end{tabular}

Table 1: Selected funds

\begin{tabular}{c|cccccccc}
\hline SR & Fund & $b_{i}^{L}$ & $b_{i}^{U}$ & $b_{w_{i}}^{L}$ & $b_{w_{i}}^{U}$ & $S R D_{i}^{L}$ & $S R D_{i}^{U}$ & $E V\left(\widetilde{S R D_{i}}\right)$ \\
\hline $\mathrm{Y}$ & $\mathrm{F} 1$ & 0.3574 & 1.795 & 0.2 & 0.4 & 0.0715 & 0.6527 & 0.3621 \\
$\mathrm{Y}$ & $\mathrm{F} 2$ & 0.295 & 1.6667 & 0.2 & 0.4 & 0.059 & 0.6061 & 0.3325 \\
$\mathrm{Y}$ & $\mathrm{F} 3$ & 0.746 & 2.727 & 0.3 & 0.5 & 0.224 & 1.736 & 0.98 \\
$\mathrm{Y}$ & $\mathrm{F} 4$ & 0.274 & 2.185 & 0.1 & 0.4 & 0.082 & 0.993 & 0.538 \\
$\mathrm{Y}$ & $\mathrm{F} 5$ & 0.25 & 1.649 & 0.3 & 0.4 & 0.025 & 0.6 & 0.312 \\
\hline $\mathrm{N}$ & $\mathrm{F} 6$ & 0.224 & 1.692 & 0 & 0 & 0 & 0 & 0 \\
$\mathrm{~N}$ & $\mathrm{~F} 7$ & 0.752 & 2.61 & 0 & 0 & 0 & 0 & 0 \\
$\mathrm{~N}$ & $\mathrm{~F} 8$ & 0.249 & 1.952 & 0 & 0 & 0 & 0 & 0 \\
$\mathrm{~N}$ & $\mathrm{~F} 9$ & 0.315 & 1.943 & 0 & 0 & 0 & 0 & 0 \\
$\mathrm{~N}$ & $\mathrm{~F} 10$ & 0.339 & 1.995 & 0 & 0 & 0 & 0 & 0 \\
\hline
\end{tabular}

Table 2: Environmental responsibility degrees

For the sake of brevity we omit the variancecovariance matrix and the vector of expected returns, but we are using weekly data from 31-122006 to 31-12-2007 provided by Morningstar Ltd. Assume we wish to select a portfolio consisting of a minimum of 3 and of a maximum of 6 funds in such a way that each non-zero weight is at least 0.05 . As upper bounds for the weights, we fix 0.25 for the first five (the socially responsible ones) and 0.15 for the conventional ones. These weights allow up to a $75 \%$ of conventional funds and up to a $100 \%$ of socially responsible funds in each feasible portfolio.

These values determine an instance of model (CP) whose efficient frontier (red curves) is shown in Figure 2. The explicit efficient frontier is described in Table 3. 


\begin{tabular}{|c|c|c|c|c|c|c|c|c|c|}
\hline$y$ & $\mathbf{a}$ & $\mathbf{b}$ & c & $r_{\min }$ & $r_{\max }$ & $\sqrt{R_{\min }}$ & $\sqrt{R_{\max }}$ & $\mathbf{L}$ & $\mathbf{U}$ \\
\hline$(0,1,0,1,1,1,1,0,0,0)$ & 41.518962 & -7.215402 & 3.632117 & 0.099653 & 0.104012 & 1.823566 & 1.825049 & \{\} & $\{2,4,6\}$ \\
\hline$(0,1,0,1,1,1,1,0,0,1)$ & 9464.790397 & -2168.389589 & 127.532271 & 0.114550 & 0.114599 & 1.826857 & 1.826863 & $\{10\}$ & $\{2,4,5\}$ \\
\hline$(0,1,0,1,1,1,1,0,0,1)$ & 5.204454 & -0.266181 & 3.299585 & 0.114599 & 0.115076 & 1.826864 & 1.826985 & $\{10\}$ & $\{2,4\}$ \\
\hline$(0,1,0,1,1,1,1,0,0,1)$ & 41.518962 & -8.624072 & 3.780483 & 0.115076 & 0.116412 & 1.826985 & 1.827346 & $\{10\}$ & $\{2,4,6\}$ \\
\hline$(0,1,0,1,1,1,1,0,0,1)$ & 9.761555 & -1.230165 & 3.350112 & 0.116412 & 0.160769 & 1.827346 & 1.845168 & & $\{2,4,6\}$ \\
\hline$(0,1,0,1,1,1,1,0,0,1)$ & 41.518962 & -11.441413 & 4.170941 & 0.160769 & 0.162011 & 1.845168 & 1.845827 & & $\{2,4,6,10\}$ \\
\hline$(0,1,0,1,1,1,0,0,1,1)$ & 10.449619 & -1.630339 & 3.400727 & 0.162011 & 0.166108 & 1.846854 & 1.848848 & $\{9\}$ & $\{2,4,6\}$ \\
\hline$(0,1,0,1,1,1,0,0,1,1)$ & 3.618703 & 0.638996 & 3.212250 & 0.166108 & 0.186736 & 1.848848 & 1.859505 & & $\{2,4,6\}$ \\
\hline$(0,1,0,1,0,1,1,0,1,1)$ & 48.649512 & -16.415042 & 4.825024 & 0.187620 & 0.189504 & 1.859505 & 1.860483 & & $\{2,4,6,7\}$ \\
\hline$(0,1,0,1,0,1,1,0,1,1)$ & 3.827972 & 0.572660 & 3.215408 & 0.189504 & 0.219765 & 1.860483 & 1.877801 & & $\{2,4,6\}$ \\
\hline$(0,1,0,1,0,1,1,0,1,1)$ & 17.114074 & -5.266986 & 3.857083 & 0.219765 & 0.230119 & 1.877801 & 1.884495 & & $\{2,4,6,9\}$ \\
\hline$(0,1,0,1,0,1,1,0,1,1)$ & 1817.414100 & -804.886017 & 92.530170 & 0.230119 & 0.231076 & 1.884495 & 1.892935 & & $\{2,6,9,10\}$ \\
\hline$(1,1,0,1,0,1,0,0,1,1)$ & 7.079298 & -2.402226 & 3.760292 & 0.231076 & 0.239175 & 1.892935 & 1.894916 & & $\{2,4,6,10\}$ \\
\hline$(1,1,0,1,0,1,0,0,1,1)$ & 16.521800 & -3.033362 & 3.371089 & 0.239175 & 0.256769 & 1.894916 & 1.918724 & & $\{2,4,9,10\}$ \\
\hline$(1,1,0,1,0,1,0,0,1,1)$ & 9.584809 & 1.514194 & 2.660776 & 0.256769 & 0.264140 & 1.918724 & 1.931183 & $\{6\}$ & $\{2,9,10\}$ \\
\hline$(1,1,0,1,0,0,0,0,1,1)$ & 7.079298 & -2.859469 & 3.990845 & 0.264140 & 0.265566 & 1.931183 & 1.931511 & \{\} & $\{2,4,10\}$ \\
\hline$(1,1,0,1,0,0,0,0,1,1)$ & 9.584809 & 1.502603 & 2.655724 & 0.265566 & 0.275246 & 1.931511 & 1.948193 & \{\} & $\{2,9,10\}$ \\
\hline$(1,1,0,1,0,1,0,0,1,1)$ & 9.584809 & 1.514194 & 2.660776 & 0.275246 & 0.276128 & 1.950307 & 1.951845 & $\{6\}$ & $\{2,9,10\}$ \\
\hline$(1,1,0,1,0,1,0,0,1,1)$ & 842.926787 & -448.629747 & 63.418568 & 0.276128 & 0.277893 & 1.951845 & 1.960133 & \{\} & $\{1,2,9,10\}$ \\
\hline$(1,1,0,0,0,1,1,0,1,1)$ & 14.767512 & -3.236231 & 3.618871 & 0.277893 & 0.278517 & 1.964678 & 1.965469 & \{\} & $\{2,6,9,10\}$ \\
\hline$(1,1,0,1,0,0,0,1,1,1)$ & 7.079298 & -2.963295 & 4.441416 & 0.278517 & 0.284024 & 2.040892 & 2.042267 & $\{8\}$ & $\{2,4,10\}$ \\
\hline$(1,1,0,1,0,0,0,1,1,1)$ & 9.584809 & 1.628508 & 2.935113 & 0.284024 & 0.303383 & 2.042267 & 2.076385 & $\{8\}$ & $\{2,9,10\}$ \\
\hline$(1,1,0,1,0,0,0,1,1,1)$ & 31.708895 & -0.012223 & 1.396550 & 0.303383 & 0.305703 & 2.076385 & 2.087140 & \{\} & $\{1,2,9,10\}$ \\
\hline$(1,1,0,0,0,1,0,1,1,1)$ & 35.526478 & -2.583291 & 1.819489 & 0.306031 & 0.360541 & 2.087140 & 2.346529 & \{\} & $\{1,2,9,10\}$ \\
\hline
\end{tabular}

Table 3: Explicit efficient frontier

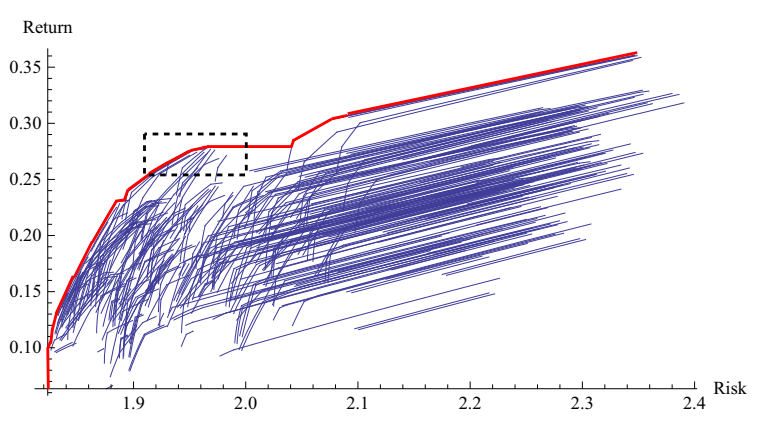

Figure 2: Efficient frontiers and subefficient portfolios

The irregular shape of the frontier is due to the fact that the function $R_{e f}(r)$ can be calculated as the pointwise minimum of the functions $R_{e f}^{y}(r)$ corresponding to the subproblems $\mathrm{CP}_{r}(y)$ resulting of fixing a specific value $y$ for the binary variables. These auxiliary subproblems are quadratic, and so their efficient frontier is a continuous piecewise parabolic curve. Furthermore, in this example, the feasible portfolios are shown in Figure 2.

On the efficient frontier, the investor can choose the zone of the risk-return plane he is interested in. Formally, this means to determine the fuzzy set $\tilde{C}$. For this, we fix $\left(r_{0}, R_{0}\right)=(0.26,1.98)$ with tolerances $\left(s_{r}, s_{R}\right)=(0.01,0.02)$ (box in Figure 2$)$. It contains just 13 subefficient portfolios and 3 portfolios which belong to the efficient frontier. This implies that each (efficient or not) feasible portfolio of any subproblem different from those appearing in the feasible region, is completely infeasible.

In order to define the fuzzy set $\tilde{E}$ determining the partially efficient portfolios, we must fix the tolerances $\left(t_{r}, t_{R}\right)$. In absence of a concrete investor to adopt a more specific criterion, a default choice could be taking as $t_{r}$ the maximum distance from a return in the efficient frontier to the minimum return in the feasible region, and analogously for $t_{R}$. In our case: $t_{r}=0.2785-0.25=0.0285$ and $t_{R}=2-1.9093=0.0907$. To determine an instance of the model (FP), we need to fix the weight $w$ for the social responsibility degree in the goal function. Let us set a quite high value, namely $w=0.8$ to favor those portfolios being quite far from the efficient frontier if they are good with regard to SRI. The obtained solutions are shown in Table 4

The parameters for the different algorithms are: (\# Individuals: 20; \# Generations: 400; Mutation prob: 0.4; Crossover prob: 0.8) for the GA in STEP 1 and (\# Individuals= 60; \# Generations: 800; Mutation prob.: 0.1; Crossover prob.: 0.85) for the GA in STEP 2. The results were obtained on a Cluster 32 cores - Intel Core i7 CPU 940 with $2.93 \mathrm{GHz}$ and $2 \mathrm{~GB}$ of memory. The CPU time for STEP 1 is 3 seconds and STEP 2 is 0.126 seconds.

The optimal solution of model (FP) is the portfolio N1 in Table 4, whose degree of membership of the decision set is 0.6250 . With this solution, the investor gets an expected return $r=0.264$, with a risk $R=1.931$ and a social responsibility degree $S R D=0.2922$.

It is interesting to compare this optimal solution with other alternatives, and therefore Table 4 contains the six best portfolios that are optimal with regard to the portfolios with the same composition. Notice that this does not mean that portfolio N2 is the second best solution of model (FP), since there are infinitely many portfolios near to $N 1$ that are better than $N 2$. What we can say is that, if we look for a portfolio with a composition different from that of $N 1$, the best possibility is $N 2$. 


\begin{tabular}{l|lllllllllllllll}
\hline & & & \multicolumn{1}{c}{ Portfolio } & & & & & $\mu_{\tilde{D}}$ SRD & return & risk \\
\hline$N 1$ & 0.205847 & 0.25 & 0 & 0.25 & 0 & 0 & 0 & 0 & 0.144153 & 0.15 & 0.6250 & 0.2922 & 0.264 & 1.931 \\
$N 2$ & 0.225809 & 0.236769 & 0.25 & 0 & 0 & 0 & 0 & 0 & 0.15 & 0.137423 & 0.6168 & 0.4055 & 0.260 & 1.980 \\
$N 3$ & 0.25 & 0.25 & 0.15 & 0.05 & 0 & 0 & 0 & 0 & 0.15 & 0.15 & 0.6091 & 0.3476 & 0.272 & 1.974 \\
$N 4$ & 0.166901 & 0.25 & 0 & 0.233099 & 0 & 0.05 & 0 & 0 & 0.15 & 0.15 & 0.5912 & 0.2690 & 0.260 & 1.924 \\
$N 5$ & 0.168049 & 0.25 & 0 & 0.231951 & 0 & 0 & 0.05 & 0 & 0.15 & 0.15 & 0.5811 & 0.2688 & 0.260 & 1.926 \\
$N 6$ & 0.25 & 0.25 & 0.15 & 0 & 0 & 0.05 & 0 & 0 & 0.15 & 0.15 & 0.5697 & 0.3207 & 0.273 & 1.978 \\
\hline
\end{tabular}

Table 4: The six best solutions for different portfolio compositions

Let us also remark that $N 1$ belongs to the efficient frontier (composition $y=(1,1,0,1,0,0,0,0,1,1)$ ). However, $\mathrm{N} 2$ is quite far from the efficient frontier but it is an efficient portfolio of its subproblem, namely, that corresponding to the composition $y=(1,1,1,0,0,0,0,0,1,1)$. And if we observe the solution $N 2$, its social responsibility grade is $S R D=0.4055$ but with a higher risk and a lower return than the values of the solution $N 1$.

In general, when applying the approach for solving an instance of model (FP), it is useful to save not only the best portfolio along the search process, but the best portfolio found for each composition. Hence, in the end we can present the investor not only the optimal portfolio, but also a list of alternatives for different compositions. These alternatives are ordered a priori according to his/her own preferences. In this way the investor is given a last chance to decide which portfolio suits better his/her preferences with regard to the trade off between risk, return and social responsibility.

\section{Conclusions}

In this paper, we have proposed a hybrid approach (named GASRP-FP) composed of two genetic algorithms to solve a fuzzy portfolio selection model with cardinality constraints, semicontinuous variable and non-financial goals. An analytical description of the efficient frontier and social responsibility solutions are obtained. This approach is more adequate for medium as well as large-sized problems. The proposed approach provides the investor not only the optimal portfolio but a list of alternatives for different compositions. In this way the investor is given a last chance to decide which portfolio suits better his/her preferences with regard to the trade off between risk, return and social responsibility.

Acknowledge - Supported by the project TIN2011-27696-C02-02 of the Ministry of Economy and Competitiveness of Spain.

\section{References}

[1] E. Ballestero, M. Bravo, B. Pérez-Gladish, M. Arenas-Parra and D. Pla-Santamaria, Socially responsible investment: A multicriteria approach to portfolio selection combining ethical and financial objectives. Eur $J$ of Operational Research, 216:487-494, 2012.
[2] A. Bilbao-Terol, M. Arenas-Parra and V. Cañal. Selection of Socially Responsible Portfolios using Goal Programming and Fuzzy Technology. Information Sciences, 189:110$125,2012$.

[3] A. Bilbao-Terol, M. Arenas-Parra and V. Cañal. A fuzzy multi-objective approach for sustainable investments. Expert Systems with Applications, 39:10904-10915, 2012.

[4] C. Calvo, C. Ivorra and V. Liern. The geometry of the efficient frontier of the portfolio selection problem. J of Financial Decision Making 7:2736, 2011.

[5] C. Calvo, C. Ivorra and V. Liern. On the computation of the efficient frontier of the portfolio selection problem. J of Applied Mathematics, 1-15, 2012.

[6] C. Calvo, C. Ivorra and V. Liern. Fuzzy portfolio selection with non-financial goals: exploring the efficient frontier. Ann of Operations Research, doi=10.1007/s10479-014-1561-2, 2014.

[7] R. Campbell, R. Huisman and K. Koedijk. Optimal Portfolio selection in a Value-at-risk framework. J of Banking \& Finance, 25:17891804, 2001.

[8] K. Deb, A. Pratap, S. Agarwal and T.A.M.T. Meyarivan. A fast and elitist multiobjective genetic algorithm: NSGA-II. IEEE T on Evolutionary Computation, 6(2):182-197, 2002.

[9] D.W. Jansen, K.G. Koedijk and C.G de Vries. Portfolio selection with limited downside risk. $J$ of Empirical Finance, 7:247-269, 2000.

[10] H.M. Markowitz. Portfolio selection. J of Finance, 7:79-91, 1952.

[11] W. Ogryczak and A. Ruszczynski. From stochastic dominance to mean-risk models: semi deviations as risk measures. Eur $J$ of $O p$ erational Research, 116:33-50, 1999.

[12] B. Pérez-Gladish, P. Méndez-Rodríguez, B. M'Zali and P. Lang. Mutual Funds Efficiency Measurement under Financial and Social Responsibility Criteria. J of Multi-Criteria Decision Analysis, 20:109-125, 2013.

[13] SIF. 2012 Report on Socially Responsible Investing Trends in the United States. Social Investment Forum: Washington DC, 2012.

[14] S. Utz, M. Wimmer, M. Hirschberger and R. Steuer. Tri-criterion inverse portfolio optimization with application to socially responsible mutual funds. Eur J of Operational Research, 134:491-498, 2014. 